10.2

\title{
Экспериментальное исследование самоорганизующегося акустического канала связи
}

\author{
(C) А.А. Невзоров, С.В. Перченко , Д.А. Станкевич \\ Волгоградский государственный университет, Волгоград, Россия \\ ๑ E-mail: perchenko@volsu.ru
}

Поступило в Редакцию 2 декабря 2020 г.

В окончательной редакции 9 декабря 2020 г.

Принято к публикации 9 декабря 2020 г.

\begin{abstract}
Приводятся результаты экспериментального исследования адаптивной системы передачи информации с фазовым разнесением. Адаптация осуществляется методами машинного обучения и заключается в регулировании фаз излучателей. В качестве среды распространения использован акустический волновод с перестраиваемой метаповерхностью. При многолучевом распространении и наличии существенной межсимвольной интерференции удалось достичь скорости передачи $1 \mathrm{kbit} / \mathrm{s}$. Время адаптации исследованной системы к резким изменениям параметров канала даже в случае значительных замираний не превышает десяти циклов обучения.
\end{abstract}

Ключевые слова: нейронная сеть, моделирование среды распространения, перестраиваемая акустическая метаповерхность.

DOI: 10.21883/PJTF.2021.06.50756.18642

Идея использования методов машинного обучения в самоорганизующихся системах передачи данных [1] или для обработки сигналов [2] не нова. Однако бурное развитие методов машинного обучения и технологий для параллельных вычислений привело к появлению новой парадигмы формирования систем связи, заключающейся в машинном синтезе алгоритмов кодирования и декодирования информации [3]. В одном из первых исследований [4] формирование способа кодирования и декодирования информации в канале связи рассматривалось как задача обучения нейронной сети типа автоэнкодер. Авторы [4] демонстрируют эффективность предлагаемого подхода по сравнению с классическими методами кодирования и декодирования информации. Однако в описанных исследованиях состояние канала связи считается известным, а его влияние на сигнал ограничивается добавлением нормального шума. Чтобы использовать все преимущества машинного обучения для реальных приложений, требуется иметь полную модель канала связи. Для решения задачи моделирования среды авторы [5] предлагают использовать условную генеративно-состязательную сеть. Такой подход хоть и позволяет частично решить проблему моделирования нестационарной среды, все же требует значительных вычислительных затрат, а также больших объемов данных для обучения. И кроме того, значительно увеличиваются время адаптации и требования к пропускной способности служебного канала передачи данных. В большинстве работ апробация методов проводится с использованием математического моделирования, а экспериментальную верификацию практически никто не проводит. В настоящей работе экспериментально исследуется самоорганизующийся канал связи с использованием метода оценивания состояния среды [6] с замираниями. В ка- честве натурной модели среды распространения используется акустический волновод с высотой канала $15 \mathrm{~mm}$ (рис. $1, a)$, стенки которого изготовлены из листового МДФ (MDF — medium density fibreboard) толщиной $10 \mathrm{~mm}$. Для уменьшения отражений на боковых гранях волновода используются звукопоглощающие клинья из вспененного пенополиэтилена.

Для изменения условий распространения в волноводе установлена перестраиваемая акустическая метаповерхность, состоящая из восьми идентичных ячеек (рис. $1, b)$. Каждая из ячеек представляет собой четыре связанных резонатора Гельмгольца, нагруженных на тонкую прямоугольную трубку. Ячейки изготовлены методом 3D-печати из PLA-пластика (плотность $1.24 \mathrm{~g} / \mathrm{cm}^{3}$ ). Объем резонаторов регулируется путем изменения их глубины перемещением подвижной вставки. При изменении глубины резонаторов в диапазоне 0-15 $\mathrm{mm}$ разность фаз $\Delta \varphi$ между акустическим давлением на входе и выходе ячейки изменяется на $2 \pi$. Это иллюстрирует рис. $1, c$, на котором представлены зависимости отношения амплитуд падающей и прошедшей волны и разности фаз от глубины резонаторов, полученные в среде моделирования COMSOL.

На одном из торцов волновода установлены 16 динамических головок CNS3508A075BER (фирмы CANOPUS). Для сигналов с длиной волны менее $20 \mathrm{~cm}$ используемая система излучателей позволяет формировать на метаповерхности волновой фронт, близкий к плоскому. Динамические головки соединены последовательно в группы по четыре, и каждая из групп подключена к выходам четырехканального усилителя мощности, собранного на микросхеме TDA7385 (фирмы ST Microelectronics). Звуковое давление внутри волновода измеряется электретным микрофоном 


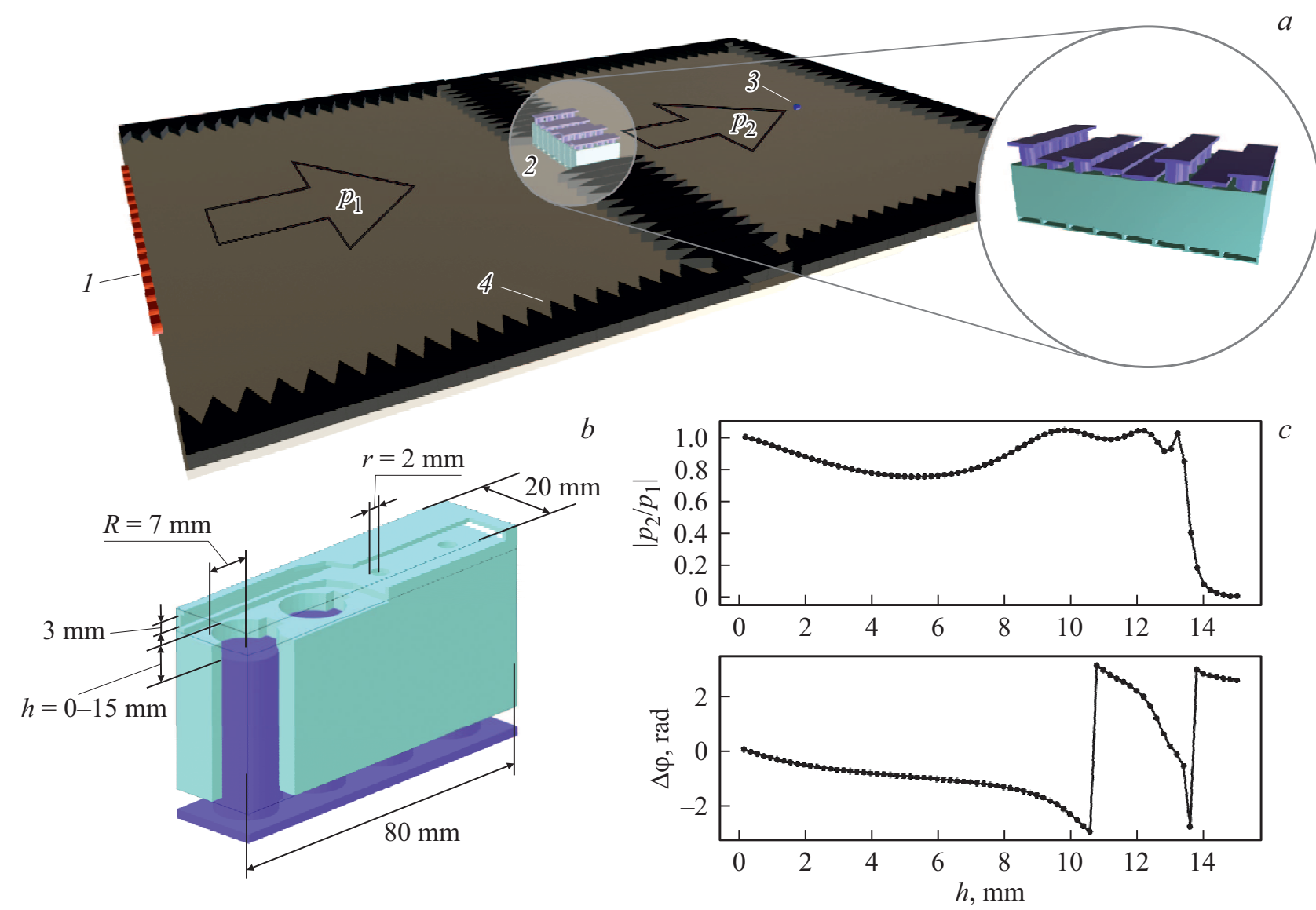

Рис. 1. $a-$ эскиз акустического волновода (верхняя стенка не показана): 1 - излучатели (16 штук), 2 - перестраиваемая метаповерхность (8 ячеек), 3 - микрофон, 4 - звукопоглощающие клинья; $b$ - ячейка перестраиваемой метаповерхности; $c$ - зависимости коэффициента передачи и разности фаз между давлениями акустической волны на входе и выходе ячейки метаповерхности от глубины резонаторов $h$.

ЕСМ-66. Микрофон и излучатели подключены к многоканальной звуковой карте UMC1820 (с частотой дискретизации до $96 \mathrm{kHz})$ фирмы Behringer.

Адаптивная система передачи информации состоит из трех искусственных нейронных сетей [6]: сеть-кодер для заданной последовательности из 5 bit формирует начальные фазы дискретных образов $s_{i}[n]=A \exp \left(-0.01(n-N / 2)^{2}\right) \cos \left(2 \pi n f / f_{0}+\varphi_{i}\right)$ излучаемых квазигармонических сигналов; сеть-имитатор среды используется в процессе адаптации для моделирования прохождения сигнала через среду; сеть-декодер преобразует принятые оцифрованные сигналы $r[n]$ в последовательность битов. Здесь $A$ - безразмерная амплитуда сигнала (при $A=1$ действующее значение напряжения на выходе звуковой карты равно $4.9 \mathrm{~V}), f=2143 \mathrm{~Hz}$ - несущая частота сигнала, $f_{0}=44.1 \mathrm{kHz}$ - частота дискретизации, $n=0-N-1$ - номер отсчета $(N=200), i=0-K-1-$ номер излучателя $(K=4)$. Все сети содержат три полносвязных слоя. Два первых слоя содержат по 200 нейронов с функцией активации гиперболический тангенс. Выходной слой сети-кодера состоит из четырех нейронов с линейной функцией активации, которые формируют начальные фазы излучаемых сигналов $\varphi_{i}$. Выходной слой сети-декодера содержит 32 нейрона с функцией активации softmax. Активация одного из этих нейронов свидетельствует о том, что передается одна из 32 возможных комбинаций из 5 bit.

Первоначально веса всех сетей задаются случайным образом по методу, описанному в работе [7]. Процесс адаптации состоит из трех этапов [6]. На первом этапе декодер обучается восстанавливать известную последовательность битов $C$ по принятому сигналу $r[n]$. Затем имитатор среды учится строить преобразование из $s_{i}[n]$ в $r[n]$. Имитатор необходим для вычисления градиентов ошибки при обучении кодера на завершающем этапе. Благодаря такому подходу удается использовать метод обратного распространения ошибки на всех этапах и тем самым увеличить производительность системы в целом. В одном цикле обучения используется $L=64$ случайно выбранных символов $C$. Поскольку на приемниках существенна межсимвольная интерференция, используются случайные перестановки символов, чтобы декодер смог верно распознавать сообщение при наложении различных сочетаний других символов. 


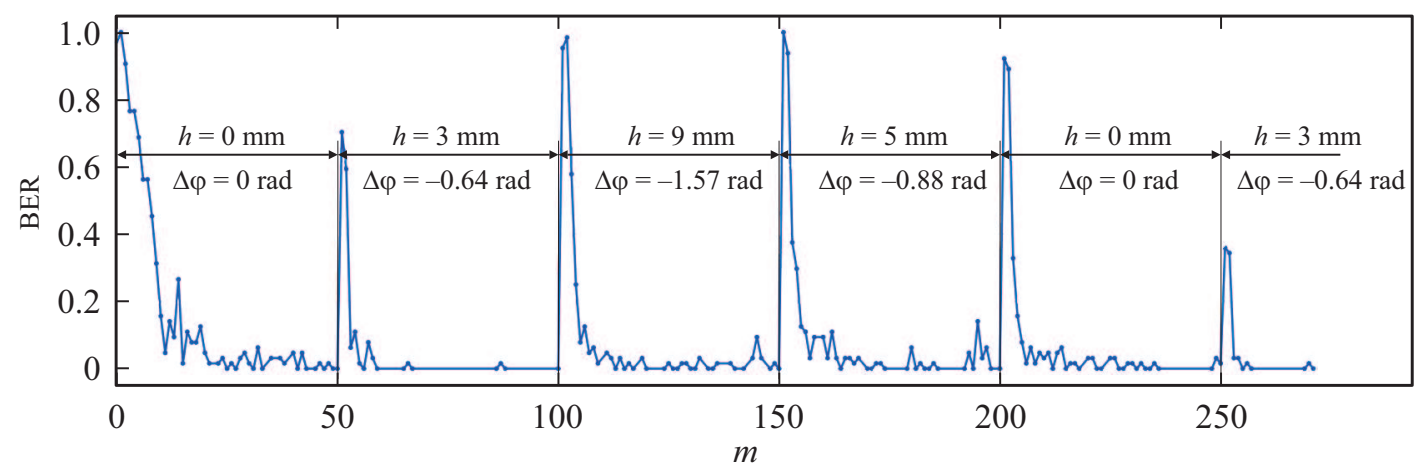

Рис. 2. Вероятность ошибки приема бита информации в процессе адаптации.

Зависимость вероятности ошибки приема бита информации (bit error rate, BER) от номера цикла обучения при различных состояниях среды распространения приведена на рис. 2. Вначале обучение системы производилось при минимальном вносимом метаповерхностью фазовом сдвиге $\Delta \varphi$, т. е. вставки всех резонаторов были полностью вдвинуты, $h=0 \mathrm{~mm}$. Примерно через 20 циклов обучения вероятность ошибки (BER) опустилась ниже 0.05 и система достигла скорости передачи $\sim 1 \mathrm{kbit} / \mathrm{s}$. Затем глубины резонаторов каждые 50 циклов последовательно изменялись $h=\{3,9,5,0,3\} \mathrm{mm}$. Моменту перестройки соответствует резкое увеличение вероятности ошибки, которая в различных случаях опускалась до уровня 0.05 за 3-10 циклов обучения. Отметим, что в исследуемом диапазоне значений $h$ отсутствует ярко выраженная корреляция между временем обучения и глубиной резонатора. Для оценки эффективности разработанного метода проводилось его сравнение с методом фазового кодирования (PSK). Установлено, что скорость передачи данных в самоорганизующейся системе всегда превосходит скорость канала, использующего классический PSK, а разница в эффективности зависит от множества параметров. Например, при $h=9 \mathrm{~mm}$ использование 32-PSK с когерентным приемом не позволяет добиться ошибки передачи символа менее $20 \%$.

Для работы описанной системы, как и для большинства адаптивных систем, требуется наличие служебного канала связи, с помощью которого осуществляется синхронизация приемника и передатчика, а также передаются значения градиентов ошибки на этапе обучения кодера. Время обучения складывается из времени передачи сообщения $C$, равного $T_{C}=L_{\tau}=L N / f_{0}=290 \mathrm{~ms}$, где $\tau$ - длительность символа, и времени обучения сетей, которое (при использовании вычислительных ресурсов графического ускорителя NVIDIA GTX680) в среднем составило $500 \mathrm{~ms}$. За один цикл по дополнительному каналу требуется передать минимум $K L$ значений градиентов ошибки; если на каждое число приходится 32 bit, то за 30 циклов обучения объем информации, переданный по дополнительному каналу, составит $30 \cdot 32 K L=245760$ bit. Видно, что накладные расходы на поддержание адаптивного канала связи в рассмот- ренном случае достаточно высоки. Однако уменьшение длительности символа $\tau$ приведет к пропорциональному росту скорости передачи, тогда как накладные расходы не изменятся. Так, для радиоканала при частоте несущей $1 \mathrm{GHz}$ и $\tau=10 \mathrm{~ns}$ можно ожидать, что скорость передачи составит не менее $0.5 \mathrm{Gbit} / \mathrm{s}$ при тех же требованиях к дополнительному каналу связи.

Проведенные нами эксперименты показали, что исследуемая система позволяет оптимизировать канал связи сразу по нескольким критериям. Например, при обучении можно ввести дополнительный штраф за увеличение амплитуды сигнала передатчика, тем самым снизив энергию, затрачиваемую передатчиком, что повысит энергоэффективность канала связи. Использованное в работе фазовое разнесенное кодирование легко можно совместить с современными методами эффективного использования полосы частот в широкополосных каналах связи. В перспективе разработанная система может полностью формировать сигналы, синтезируя новые методы кодирования в каналах связи с любой доступной шириной полосы пропускания. Кроме того, имеется потенциал для минимизации времени обучения системы в целом, этого можно добиться за счет предобучения или оптимизации архитектур отдельных сетей под конкретную задачу.

\section{Финансирование работы}

Исследование выполнено за счет гранта Российского научного фонда (проект № 19-79-00098).

\section{Конфликт интересов}

Авторы заявляют, что у них нет конфликта интересов.

\section{Список литературы}

[1] M. Ibukahla, J. Sombria, F. Castanie, N.J. Bershad, IEEE Trans. Commun., 45 (7), 768 (1997). DOI: 10.1109/26.602580

[2] A. Fehske, J. Gaeddert, J.H. Reed, Proc. IEEE Int. Symp. on new frontiers in dynamic spectrum access networks (DYSPAN) (Baltimore, MD, USA, 2005), p. 144.

DOI: 10.1109/DYSPAN.2005.1542629 
[3] T. Wang, C.K. Wen, H. Wang, F. Gao, T. Jiang, S. Jin, Chin. Commun., 14 (11), 92 (2017). DOI: 10.1109/CC.2017.8233654

[4] T. O’Shea, J. Hoydis, IEEE Trans. Cognit. Commun. Netw., 3 (4), 563 (2017). DOI: 10.1109/TCCN.2017.2758370

[5] H. Ye, G. Li, B.H.F. Juang, K. Sivanesan, arxiv: 1807.00447 (2018).

[6] А.А. Невзоров, А.А. Орлов, Д.А. Станкевич, Письма в ЖТФ, 45 (14), 7 (2019).

DOI: $10.21883 /$ PJTF.2019.14.48013.17836

[7] X. Glorot, Y. Bengio, in Proc. of the 13th Int. Conf. on artificial intelligence and statistics. JMLR Workshop Conf. Proc., 9, 249 (2010). 